\section{PTU-085 DECOMPENSATED ALCOHOLIC LIVER DISEASE (ALD): HIGH LONG-TERM MORTALITY DESPITE INITIAL SURVIVAL}

doi:10.1136/gutjnl-2013-304907.177

1." A N Aljoudeh, 'E McFarlane, 'M Karajeh, 'D Gleeson. 'Hepatology, Sheffield Teaching Hospitals Foundation NHS Trust, Sheffield, UK

Introduction Whilst early management and outcome of decompensated ALD has been extensively studied, there are few published data on long-term outcome. We have previously (McFarlane, Gut 2006; 55:A2 and 55:A36) reported on early and 4-yr mortality in 249 patients (163 men, age (mean(range)) 50(27-77) yr) admitted consecutively to our unit between 1/4/1998 and 31/12/2005 with first presentation of decompensated ALD (Child grade B or C). Here we aimed to assess long-term mortality and its associations in this cohort.

Methods We reviewed available hospital records, and death certificates and contacted surviving patients and general practitioners to assess who had died, the causes of death and the patients' overall alcohol drinking behaviour subsequent to the index hospital episode (classified as: abstinent, continued drinking but reduced, and did not reduce).

Results 37 patients died during the index hospital episode, all because of liver disease. The other 212 patients (including one transplanted during the index episode) were followed up for 4.3 (0.0313.0) years. Only one other patient was transplanted. 154 patients have subsequently died. Cause of death is known in 134 (87\%) and was due to liver disease in 95 (71\%) of these. Only 4 patients died of hepatocellular carcinoma. Overall 5- and 10- year total mortality rates were $52+(\mathrm{SEM}) 4 \%$ and $75+3 \%$ respectively; corresponding rates from causes known to be liver related were $41+5 \%$ and $51+4 \%$. Patients who were abstinent $(n=52)$ had lower total and known liver-related mortality $(61+9 \%$ and $20+6 \%$ after $10 y r)$ compared to those who continued but reduced ( $n=105 ; 73+5 \%$ $\mathrm{p}=0.122$ and $53+6 \% \mathrm{p}=0.013$ ) and to those who did not reduce $(\mathrm{n}=53 ; 91+4 \% \mathrm{p}<0.001$ and $71+7 \% \mathrm{p}<0.001)$. In Cox regression analysis, both total and known liver-related mortality were independent of age, gender and severity of liver dysfunction at index presentation (Child, MELD, Glasgow and Maddrey scores) but were strongly associated with subsequent drinking behaviour (both $\mathrm{p}<$ 0.001 ) and inversely associated with serum albumin at discharge following index hospital episode ( $p=0.001$ and 0.019 ).

Conclusion Patients with decompensated ALD who survive their first hospital episode have high long-term mortality, mainly due to liver disease, which is reduced but not prevented by abstinence.

Disclosure of Interest None Declared

\section{PTU-086 OUTCOME OF PATIENTS WITH ALCOHOL LIVER DISEASE ADMITTED TO INTENSIVE CARE IN A TERTIARY REFERRAL CENTRE - SINGLE CENTRE EXPERIENCE}

doi:10.1136/gutjnl-2013-304907.178

1."A Staicu, ${ }^{2} \mathrm{P}$ Jackson, 'S Everett. 'Gastroenterology; ${ }^{2}$ Intensive Care Department, Leeds Teaching Hospital, Leeds, UK

Introduction Hospital admissions with decompensated chronic alcoholic liver disease (ALD) have been increasing, leading to increased pressure on intensive care unit (ICU) services. We aimed to determine the outcome and prognostic factors for patients with ALD requiring admission to ICU.

Methods This was a retrospective study over a five year period (January 2006 - December 2010) of ICU admissions with ALD to St James' Hospital Leeds, either known or at first presentation. We reviewed in detail case notes and the laboratory database based on a pre-established proforma. ICU and hospital mortality were recorded along with outcome in diagnostic and organ support subgroups.
Results 29 patients with ALD admitted to ICU were included, median age 46 yrs, 79\% males. 82.7\% (24/29) were Child Pugh Score C. Mean ITU stay was 5.2 days. Overall ICU and hospital mortality was $51.7 \%(15 / 29)$ and $65.5 \%(19 / 29)$ respectively. $20(69 \%)$ were previously known ALD and $9(31 \%)$ were at first presentation. ICU and in hospital mortality in first presentation ALD group was $67 \%$ $(6 / 9)$ and $89 \%$ (8/9) compared with 45\% (9/20) and 55\% (11/20) respectively for previously known ALD. The reasons for admission to ICU were variceal bleeding (59\%), sepsis $(17 \%)$ and $37 \%$ other (encephalopathy, other causes of bleeding, seizures, renal support). ICU and in hospital mortality in the variceal bleeding subgroup was $47 \%$ and $58 \%$ respectively compared to $100 \%$ ICU and hospital mortality for sepsis. Patients with multiorgan failure had the worst prognosis. Organ failure was predefined based on level of support required on admission to ITU.

Abstract PTU-086 Table 1 Mortality in organ failure subgroup

\begin{tabular}{llll}
\hline Number of organ failure & $\mathbf{n}$ & ICU Mortality $\mathbf{n}(\%)$ & Hospital Mortality $\mathbf{n}(\%)$ \\
\hline 1 & 5 & $1 / 5(20)$ & $3 / 5(60)$ \\
2 & 4 & $1 / 4(25)$ & $2 / 4(50)$ \\
3 & 13 & $8 / 13(61)$ & $12 / 13(92)$ \\
4 & 7 & $5 / 7(71)$ & $7 / 7(100)$ \\
\hline
\end{tabular}

Conclusion Mortality remains high in ALD patients admitted to ICU. In this study, acute variceal bleeding and single or dual organ support were associated with better survival outcomes compared with other presentations and outcomes were not better in patients presenting for the first time with ALD compared to recurrent admissions. Escalation therapy to ICU for patients with sepsis or requiring multiple organ support may be futile.

Disclosure of Interest None Declared

\section{REFERENCES}

1. J. Mackle, D. G. Swann and B. Cook, One year outcome of intensive care patients with decompensated alcoholic liver disease, British Journal of Anaesthesia 97 (4): 496-8 (2006)

2. P Jackson, D Gleeson, Alcohol Liver Disease, Contin Educ Anaesth Crit Care Pain (2010) 10 (3): 66-71

3. S. J. Thomson, C. Moran, M. L. Cowan, S. Musa, R. Beale, D. Treacher, M. Hamilton, R. M. Grounds, T. M. Rahman Outcomes of Critically III Patients with Cirrhosis Admitted to Intensive Care: An Important Perspective from the Non-transplant

\section{PTU-087 WITHDRAWN BY AUTHOR}

\section{PTU-088 VARIATION IN PRACTICE OF MANAGEMENT OF SPONTANEOUS BACTERIAL PERITONITIS (SBP)}

doi:10.1136/gutjnl-2013-304907.179

1.*A Sugumaran, ${ }^{1} \mathrm{C}$ Ch'ng. 'Gastroenterology and hepatology, Singleton hospital, Swansea, UK

Introduction Spontaneous Bacterial Peritonitis (SBP) is the most frequent and life-threatening infection in patients with liver cirrhosis, requiring prompt recognition and treatment. Clinical practise guidelines on management of SBP were released by EASL in $2010^{1}$ and AASLD in $2009^{2}$. We wanted to determine if there is uniformity in SBP management in hospitals in Wales, UK.

Methods Invitation to online survey was sent via email link to all WAGE members (Welsh Association for Gastroenterologists and Endoscopists). 25 members responded and results analysed. There were total of 10 questions with focus on diagnosis and drug treatment.

Results $76 \%$ of respondents would test for SBP in any cirrhotic ascites including day case routine admissions for therapeutic paracentesis; $12 \%$ do not test for day-case asymptomatic patients. $12 \%$ 